\title{
Epidemiology of Intracranial Hemorrhage Associated with Oral Anticoagulants in Spain: Trends in Anticoagulation Complications Registry - The TAC 2 Study
}

\author{
Gustavo Zapata-Wainberg $^{\text {a }}$ Sonia Quintas ${ }^{b}$ \\ Álvaro Ximénez-Carrillo Rico ${ }^{a}$ Jaime Masjuán Vallejo ${ }^{c}$ Pere Cardona ${ }^{d}$ \\ Mar Castellanos Rodrigo e Lorena Benavente Fernández ${ }^{f}$ \\ Andrés García Pastorg José Egido ${ }^{\mathrm{h}}$ José Maciñeiras ${ }^{\mathrm{i}}$ \\ Joaquín Serenaj María del Mar Freijo Guerrerok Francisco Monichel \\ José Vivancos ${ }^{a}$ TAC 2 Study Investigators \\ ${ }^{a}$ Hospital Universitario de La Princesa, Instituto de Investigación Sanitaria La Princesa, \\ Madrid, Spain; ${ }^{b}$ Hospital Universitario de La Princesa, Madrid, Spain; ${ }^{c}$ Hospital Universitario \\ Ramón y Cajal, Madrid, Spain; 'Hospital Universitari de Bellvitge, L'Hospitalet de Llobregat,

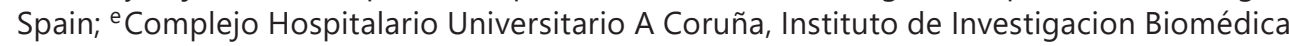 \\ A Coruña, Coruña, Spain; ${ }^{f}$ Hospital Universitario Central de Asturias, Oviedo, Spain; \\ gHospital General Universitario Gregorio Marañón, Madrid, Spain; hospital Clínico \\ Universitario San Carlos, Madrid, Spain; 'Complexo Hospitalario Universitario de Vigo, \\ Vigo, Spain; ${ }^{j}$ Hospital Universitario Dr. Josep Trueta, Girona, Spain; ${ }^{k}$ Hospital de Basurto, \\ Montevideo Etorb, Bilbao, Spain; 'Hospital Universitario Virgen del Rocío, Seville, Spain
}

\section{Keywords}

Direct oral anticoagulants $\cdot$ Incidence $\cdot$ Intracranial hemorrhage $\cdot$ Neuroepidemiology $\cdot$ Oral anticoagulants · Vitamin $\mathrm{K}$ antagonists

\begin{abstract}
Objective: Patients receiving treatment with oral anticoagulants (OACs) are at risk of intracranial hemorrhage $(\mathrm{ICH})$. In this study, we describe the epidemiological and clinical characteristics of patients receiving OACs who experience $\mathrm{ICH}$ and compare those receiving vitamin $\mathrm{K}$ antagonists (ICH-VKAs) with those receiving direct OACs (ICH-DOACs). Methods: We performed a national, multicenter, descriptive, observational, retrospective study of all adult patients receiving OACs who were admitted to the neurology department with $\mathrm{ICH}$ over a 1-year period. The study population was divided into 2 groups (ICH-VKAs and ICH-DOACs). Epide-
\end{abstract}

A complete list of the study investigators can be found in the Appendix.

Gustavo Zapata-Wainberg, MD

Hospital Universitario de La Princesa

Diego de León St. \#62, 7th Fl.

ES-28006 Madrid (Spain)

E-Mail gzapatawainberg @ gmail.com 
miological, clinical, radiological, and therapy-related variables, as well as functional outcome, were compared at 3 months. A total of 366 cases were included $(331 \mathrm{ICH}-V K A s, 35 \mathrm{ICH}-$ DOACs). Results: The crude annual incidence of OAC-induced ICH was $3.8(95 \% \mathrm{Cl}, 2.78-3.41)$ per 100,000 inhabitants/year. The mean $( \pm S D)$ age was greater for ICH-DOACs ( $81.5 \pm 8.3$ vs. $77.7 \pm 8.3$ years; $p=0.012$ ). The median (IQR) volume of the hemorrhage was lower for ICHDOACs (11 [30.8] vs. 25 [50.7] $\mathrm{mL} ; p=0.03$ ). The functional independence rate at 3 months (modified Rankin Scale, mRS $<3$ ) was similar in both groups, although stroke-related mortality was greater in ICH-VKAs (40 vs. $72.7 \% ; p=0.02$ ). The most frequently indicated poststroke antithrombotic therapy was DOACs (38.7\%). Conclusion: We found that the incidence of OACinduced $\mathrm{ICH}$ was greater than in previous studies. Hemorrhage volume and mortality were lower in ICH-DOACs than in ICH-VKAs. After stroke, DOACs were the most frequently indicated antithrombotic treatment.

(c) 2018 S. Karger AG, Basel

\section{Introduction}

The mean annual risk of ischemic stroke in patients with atrial fibrillation (AF) not treated with anticoagulants is $6-18 \%[1,2]$. Vitamin K antagonists (VKAs) have traditionally been the first choice for prophylaxis and have reduced the risk of stroke in patients with AF by approximately $67 \%$ [1].

The beneficial effect of VKAs can be diminished by the increased risk of hemorrhage. The risk of intracranial hemorrhage (ICH) in patients receiving VKAs is $0.3-3 \%$, i.e., an 8- to 10 -fold greater risk than in patients who do not take VKAs $[3,4]$. Furthermore, not only is the risk of ICH increased, but once the hemorrhage occurs, the initial volume [5], risk of expansion, severity, and probability of death are also higher [6, 7]. The mortality rate of ICH is $40-67 \%$ $[3,4,6]$, and the probability of functional recovery is $17-24.3 \%$ [3].

Compared with VKAs, the new direct oral anticoagulants (DOACs) [8-14] have been proven to improve or at least equal the reduction in the risk of ischemic stroke/systemic embolism, reduce the risk of ICH by up to $30-70 \%$, and present lower mortality rates, owing mainly to the reduction in fatal bleeds $[2,4]$. Other advantages of DOACs are the few associated drug and food interactions, the rapid onset of action, and the fact that anticoagulation does not have to be monitored [15].

The objective of this study was to perform an exhaustive analysis of the epidemiological, clinical, and radiological characteristics and functional outcome of patients receiving treatment with OACs who experience ICH and to make a comparison between ICH-VKAs and ICH-DOACs.

\section{Patients and Methods}

Design

We performed a multicenter, observational, retrospective study based on prospective registers of consecutive patients diagnosed with stroke in most Spanish stroke units. The units were representative of most of the autonomous communities of Spain. The objective of the study was to perform an exhaustive analysis of the epidemiological, clinical, and radiological characteristics and functional outcome of patients receiving treatment with OACs who experience ICH and to make a comparison between ICH-VKAs and ICHDOACs. The inclusion criteria were diagnosis of spontaneous ICH (intraparenchymal and/or intraventricular), age $\geq 18$ years, current treatment with an OAC, and admission to the neurology department (stroke unit or general neurology) during the study period. We excluded cases of subarachnoid hemorrhage, epidural hematoma, and subdural hematoma (irrespective of whether they were traumatic or spontaneous). The 
participating centers had to fulfill the following requirements: general hospital status, well-established catchment area, 24/7 availability of an emergency department with neuroimaging (cerebral computed tomography), neurology department with a stroke unit, and a prospective database of patients who had experienced a stroke.

Data were collected via an online form where each center uploaded their findings for demographic variables, vascular risk factors, anticoagulant treatment, characteristics of the stroke, bleeding complications during admission, radiology findings, length of stay, and short- and long-term treatment. Functional outcome at 3 months was measured using the modified Rankin Scale (mRS); this evaluation was performed in person by a neurologist and functional independence was considered when $\mathrm{mRS}<3$. Patients with a previous $\mathrm{mRS}$ score $\geq 3$ (patients who were previously dependent) were excluded from the analysis of functional status at 3 months in order to avoid assigning subsequent dependence to a preexisting condition. International normalized ratio (INR) was measured at admission and the therapeutic range was considered 2-3 in all patients (except for those bearing metallic cardiac valves in which the therapeutic range was considered to be between 2.5 and 3.5). ICH volume was measured by the investigators of each site and extensive hemorrhage was defined as $>100 \mathrm{~mL}$, involvement of deep and lobar areas, and undetermined anatomical origin. In order to perform the epidemiological analysis, we collected data on the activity at each of the participating centers during the study period: catchment population, total number of patients admitted to the neurology department, and total number of patients admitted with a diagnosis of acute stroke (ischemic and hemorrhagic).

We included patients admitted from January 1 to December 31, 2015. The study was performed over a natural year in order to avoid seasonal variations.

\section{Statistical Analysis}

Quantitative variables were assessed using measures of central tendency and dispersion depending on the distribution and normality of the variables. Normally distributed variables were expressed as mean $\pm \mathrm{SD}$; nonnormally distributed variables were expressed as median (IQR). Variables were compared using the $t$ test or Mann-Whitney test depending on whether the variables fulfilled the criteria for each test. The qualitative variables were expressed as percentages between groups, and associations were analyzed using the $\chi^{2}$ test (significance was adjusted in multiple comparisons using the Bonferroni correction). Statistical significance was set at $p<0.05$. Data were analyzed using IBM SPSS ${ }^{\circledR}$ Statistics for Windows, Microsoft Excel ${ }^{\circledR} 2016$, R v3.3.3 [16], and OpenEpi [17].

\section{Results}

Description of the Sample: Clinical and Epidemiological Characteristics

The study population comprised 366 patients from 29 hospital stroke units throughout Spain (Fig. 1). The hospitals attended a population of $11,864,971$ inhabitants $(25 \%$ of the population of Spain during the year of the study); therefore, the crude incidence of OACinduced ICH was 3.8 (95\% CI, 2.78-3.41) cases per 100,000 inhabitants. OAC-induced ICH accounted for $18.2 \%$ of the total admissions for ICH, and ICH-DOACs accounted for $1.7 \%$ of these admissions (Table 1). Of the 366 cases of OAC-induced ICH, 35 (9.5\%) involved ICH-DOACs. Table 2 summarizes the clinical characteristics of both groups and highlights that ICH-DOACs had a higher mean age ( $81.5 \pm 8.3$ vs. $77.7 \pm 8.3$ years; $p=0.012)$ and more often had a history of ischemic stroke (34.3 vs. 19.95; $p=0.049$ ). Stroke seemed to be more severe in ICH-VKAs than in ICH-DOACs (median National Institutes of Health Stroke Scale [NIHSS], 9 [14] vs. 7 [12]), although the difference was not statistically significant ( $p=$ $0.37)$.

\section{Data on Previous Treatment}

The median (IQR) duration of treatment was greater for ICH-VKAs (36 [57.5] vs. 12 [7.75] months; $p<0.001$ ). The mean INR in the ICH-VKA group was $2.7 \pm 1.1$; the INR was supratherapeutic in only $28.7 \%$ of patients in this group. There were no differences in aPTT 


\section{Interventional Neurology}

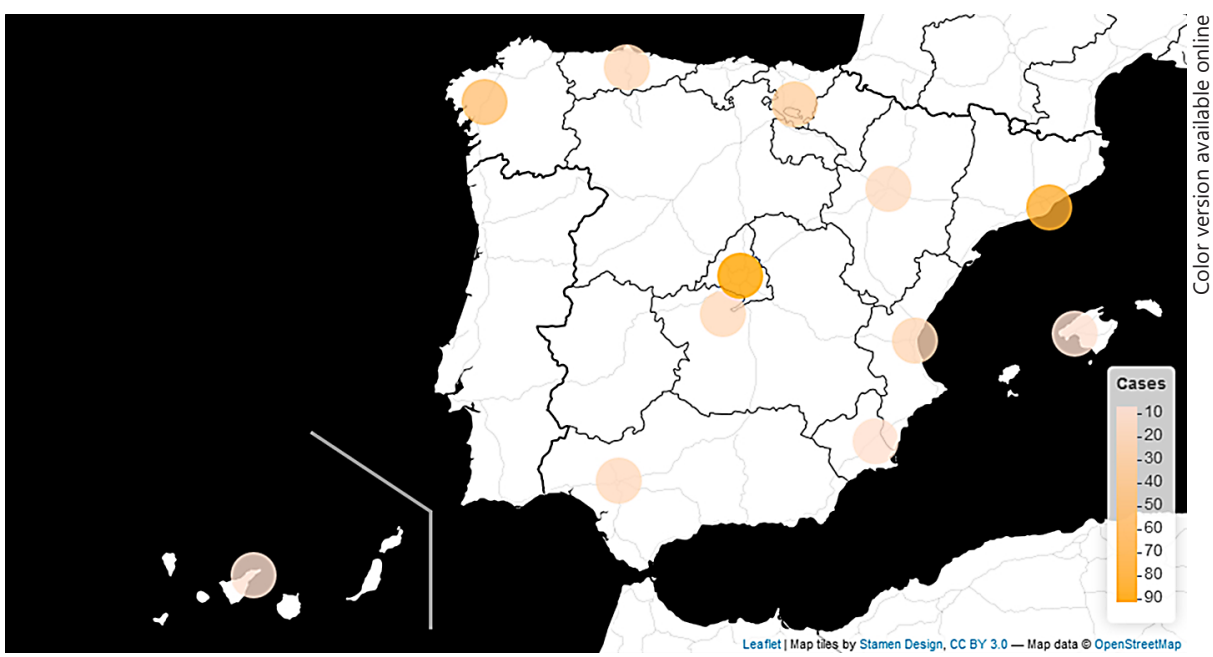

Fig. 1. Geographical distribution of cases: the dots show the location of the main city in the autonomous region.

Table 1. Epidemiological characteristics of ICHs by group

\begin{tabular}{llll}
\hline & $\begin{array}{l}\text { VKA } \\
(n=331)\end{array}$ & $\begin{array}{l}\text { DOAC } \\
(n=35)\end{array}$ & $\begin{array}{l}\text { Total } \\
(n=366)\end{array}$ \\
\hline $\begin{array}{l}\text { Crude incidence } \times 100,000 \text { inhabitants/ } \\
\text { year }(95 \% \mathrm{CI})\end{array}$ & $2.79(2.5-3.1)$ & $0.3(0.20-0.40)$ & $3.085(2.78-3.41)$ \\
$\begin{array}{l}\text { \% with respect to total admissions to } \\
\text { the neurology department }\end{array}$ & 1.2 & 0.1 & 2.3 \\
$\begin{array}{l}\% \text { with respect to the total number of } \\
\text { cases of stroke }\end{array}$ & 2.0 & 0.2 & 18.2 \\
\hline $\begin{array}{l}\text { \% with respect to the total number of } \\
\text { cases of hemorrhage }\end{array}$ & 16.4 & 1.7 & 2.3 \\
\hline
\end{tabular}

ICH, intracranial hemorrhage; DOAC, direct oral anticoagulant; VKA, vitamin K antagonist.

between the groups (ICH-VKA: $48.7 \pm 52 \mathrm{~s}$ vs. ICH-DOACs: $49.7 \pm 66.4 \mathrm{~s} ; p=0.9$ ). We found no significant differences between the groups with respect to concomitant treatment (contraceptives, antiepileptic drugs, digoxin, aspirin, clopidogrel, and nonsteroidal anti-inflammatory drugs).

\section{Radiological Characteristics}

Median (IQR) hemorrhage volume was significantly lower in ICH-DOACs than in ICH-VKAs (25 [50.7] vs. 11 [30.8] mL; $p=0.03$ ). The topographical distribution of the hemorrhages was comparable in both groups, although the absence of extensive hemorrhaging was noteworthy in the ICH-DOAC group (Table 3). Similarly, in the ICH-DOAC group, we found a lower frequency of ventricular involvement because of the absence of primary intraventricular hemorrhage in ICH-DOACs compared with ICH-VKAs (3.3\%). 
Table 2. Clinical characteristics of the sample

\begin{tabular}{|c|c|c|c|}
\hline & \multicolumn{2}{|c|}{ Type of oral anticoagulant } & \multirow[t]{2}{*}{$p$ value } \\
\hline & $\operatorname{VKA}(n=331)$ & $\operatorname{DOAC}(n=35)$ & \\
\hline Female sex & $129(39.00)$ & $18(51.40)$ & 0.153 \\
\hline Age (mean $\pm S D$ ), years & $77.7 \pm 8.3$ & $81.5 \pm 8.3$ & 0.012 \\
\hline Baseline NIHSS, median (IQR) & $9(14)$ & $7(12)$ & 0.336 \\
\hline Prestroke functional independence ( $\mathrm{mRS}<3$ ) & $270(81.60)$ & $24(68.60)$ & 0.066 \\
\hline Arterial hypertension & $265(80.10)$ & $30(85.70)$ & 0.421 \\
\hline Diabetes mellitus & $113(34.10)$ & $9(25.70)$ & 0.315 \\
\hline Hyperlipidemia & $160(48.30)$ & $13(3710)$ & 0.207 \\
\hline Ischemic heart disease & & $13(37.10)$ & 0.068 \\
\hline Atrial fibrillation & $59(17.80)$ & $2(5.70)$ & 0.748 \\
\hline Previous ischemic stroke & $263(79.50)$ & $27(77.10)$ & 0.049 \\
\hline Previous hemorrhagic stroke & $66(19.90)$ & $12(34.30)$ & 0.064 \\
\hline Leukoaraiosis on CT or MRI & $9(2.70)$ & $3(8.60)$ & 0.192 \\
\hline Smoking & $48(14.50)$ & $8(22.90)$ & 0.108 \\
\hline Alcohol consumption & $39(11.80)$ & $1(2.90)$ & 0.135 \\
\hline Anticoagulant drug & $20(6.00)$ & $0(0.00)$ & \\
\hline Acenocoumarol & $303(91.50)$ & & - \\
\hline Warfarin & $28(8.50)$ & - & - \\
\hline \multirow[t]{2}{*}{ Dabigatran } & - & - & - \\
\hline & - & $8(22.90)$ & \\
\hline Rivaroxaban & - & $17(48.60)$ & - \\
\hline Apixaban & & $10(28.60)$ & - \\
\hline \multicolumn{4}{|l|}{ OAC indication (other than atrial fibrillation) } \\
\hline Pulmonary embolism & $13(3.90)$ & $1(2.90)$ & 0.7 \\
\hline Deep venous thrombosis & $11(3.30)$ & $0(0)$ & 0.6 \\
\hline Thrombophilia & $3(0.90)$ & $0(00)$ & 1 \\
\hline Metallic prosthetic cardiac valves & $16(4.8)$ & $0(0)$ & 0.4 \\
\hline
\end{tabular}

Values are $n(\%)$ unless otherwise indicated. DOAC, direct oral anticoagulant; IQR, interquartile range; mRS, modified Rankin Scale; NIHSS, National Institutes of Health Stroke Scale; VKA, vitamin K antagonist.

Table 3. Topographical distribution of hemorrhages by treatment group

\begin{tabular}{lccc}
\hline & \multicolumn{2}{l}{ Type of anticoagulant } & \\
\cline { 2 - 3 } & VKA & DOAC & 0.31 \\
\hline Lobar & $113(34.10)$ & $9(25.70)$ & 0.061 \\
Deep & $153(46.20)$ & $22(62.90)$ & 0.96 \\
Brainstem & $9(2.70)$ & $1(2.90)$ & 0.75 \\
Cerebellum & $34(10.30)$ & $3(8.60)$ & 0.23 \\
Extensive hemispheric & $18(5.40)$ & $0(0.00)$ & 0.03 \\
Intraventricular (primary or associated) & $81(24.50)$ & $3(8.60)$ & \\
\hline
\end{tabular}

Values are $n(\%)$. DOAC, direct oral anticoagulant; VKA, vitamin K antagonist. 
Table 4. Hemorrhagic complications, acute-phase treatments, length of stay, and functional outcomes at 3 months

\begin{tabular}{|c|c|c|c|}
\hline & \multicolumn{2}{|c|}{ Type of anticoagulant } & \multirow[t]{2}{*}{$p$} \\
\hline & VKA & DOAC & \\
\hline \multicolumn{4}{|l|}{ Bleeding complications } \\
\hline Upper digestive tract bleeding & $2(0.60)$ & $0(0.00)$ & 0.64 \\
\hline Lower digestive tract bleeding & $3(0.90)$ & $0(0.00)$ & 0.57 \\
\hline Hematuria & $1(0.30)$ & $0(0.00)$ & 0.74 \\
\hline Hemoptysis & $1(0.30)$ & $0(0.00)$ & 0.74 \\
\hline Epistaxis & $2(0.60)$ & $0(0.00)$ & 0.64 \\
\hline \multicolumn{4}{|l|}{ Treatment administered } \\
\hline Decompressive craniectomy & $5(1.50)$ & $0(0.00)$ & 0.46 \\
\hline Drainage of hematoma & $7(2.10)$ & $0(0.00)$ & 0.38 \\
\hline External ventricular drain & $19(5.70)$ & $1(2.90)$ & 0.47 \\
\hline Minimally invasive surgery & $0(0.00)$ & $0(0.00)$ & - \\
\hline Vitamin $\mathrm{K}$ & $200(60.40)$ & $3(8.60)$ & $<0.001$ \\
\hline Hemodialysis & $4(1.20)$ & $0(0.00)$ & 0.51 \\
\hline Fresh frozen plasma & $9(2.70)$ & $1(2.90)$ & 0.96 \\
\hline Prothrombin complexes & $162(48.90)$ & $8(22.90)$ & 0.03 \\
\hline Monoclonal antibodies (reversal agents) & $0(0.00)$ & $0(0.00)$ & - \\
\hline Withdrawing/withholding therapy & $60(18.10)$ & $3(8.60)$ & 0.15 \\
\hline Stay in ICU during admission & $44(13.30)$ & $1(2.90)$ & 0.07 \\
\hline \multicolumn{4}{|l|}{ Length of stay (mean \pm SD), days } \\
\hline In the stroke unit & $2.19 \pm 2.6$ & $1.7 \pm 2.5$ & 0.35 \\
\hline Total length of stay & $12.49 \pm 13.7$ & $10.9 \pm 11$ & 0.49 \\
\hline \multicolumn{4}{|l|}{ Outcome at 3 months ${ }^{\mathrm{a}}$} \\
\hline Functional independence $(\mathrm{mRS}<3$ ) & $85(31.50)$ & $10(42.90)$ & 0.28 \\
\hline Death & $113(42.00)$ & $7(28.60)$ & 0.06 \\
\hline Cause of death (all patients) & $101(72.70)$ & $4(40.00)$ & \\
\hline Stroke-related & & & 0.02 \\
\hline Other cardiovascular causes & $7(5.00)$ & $0(0.00)$ & 0.5 \\
\hline Other & $19(13.70)$ & $5(50.00)$ & 0.002 \\
\hline Unknown & $12(8.60)$ & $1(10.00)$ & 0.88 \\
\hline
\end{tabular}

Values are $n(\%)$ unless otherwise indicated. DOAC, direct oral anticoagulant; ICU, intensive care unit; mRS, modified Rankin scale; VKA, vitamin K antagonist. ${ }^{a}$ Only previously independent patients.

\section{In-Hospital Outcome and Medium-Term Outcome}

The overall rate of hemorrhagic complications was low, the most frequent type being upper digestive tract bleeding, which affected $0.9 \%$ of ICH-VKAs (Table 4). No systemic bleeding events were detected in ICH-DOACs during admission. The distribution of treatment administered during the acute phase was similar in both groups, although it is noteworthy that in ICH-DOACs, no patients underwent surgery and the frequency of admission to the intensive care unit was lower. Administration of vitamin Kand prothrombin complexes was more common in ICH-VKAs. The mean total length of stay seemed to be greater - albeit without statistical significance - in ICH-VKAs (12.49 \pm 13.7 vs. $10.9 \pm 11$ days; $p=0.35)$, as was the need to remain in the stroke unit ( $2.19 \pm 2.6$ vs. $1.7 \pm 2.5$ days; $p=0.49$ ). There were no statistically significant differences in functional status between ICH-DOACs and ICH-VKAs groups at 3 months (mRS $<3$ at 3 months: 42.9 vs. $31.5 \%$ respectively; Fig. 2) 


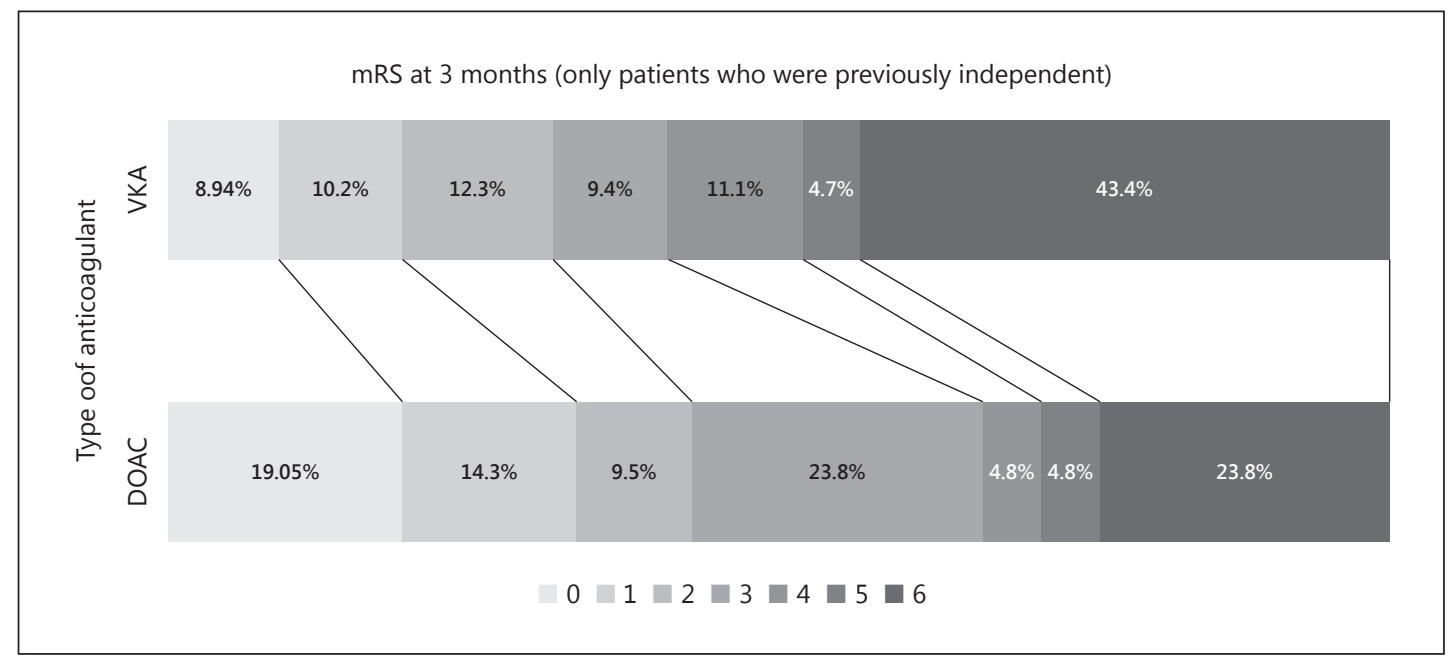

Fig. 2. Functional outcome (modified Rankin Scale, mRS) of patients at 3 months after stroke. DOAC, direct oral anticoagulant; VKA, vitamin K antagonist.

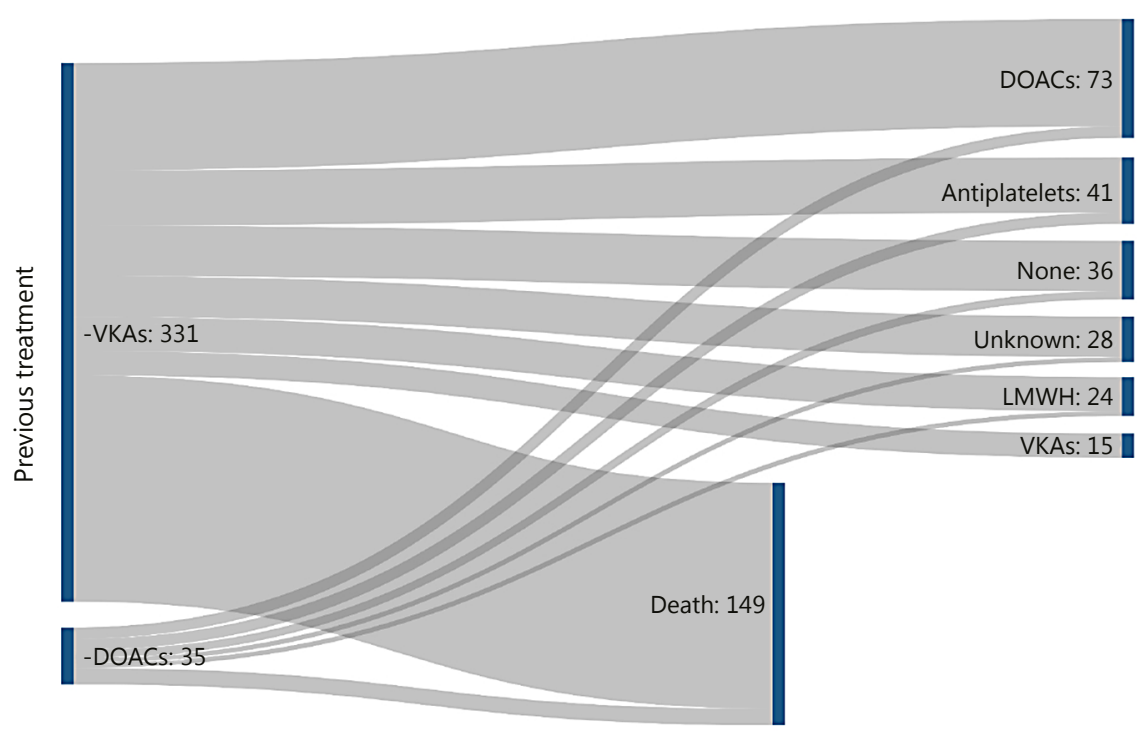

Fig. 3. Antithrombotic treatment at 3 months according to the type of previous treatment. DOACs, direct oral anticoagulants; VKAs, vitamin K antagonists; LMWH, low molecular-weight heparin.

More patients died in the ICH-VKA group, and the most frequent cause of death was cerebral hemorrhage; stroke-related mortality was greater in ICH-VKAs (40 vs. 72.7\%; $p=$ 0.02). The most frequently indicated treatment in survivors was DOACs (33.6\%), followed by antiplatelet agents (18.9\%), and then by withholding of antithrombotic treatment $(16.6 \%)$ (Fig. 3). 


\section{Discussion}

In our study, we found the incidence of OAC-induced ICH to be 3.8 cases per 100,000 inhabitants, i.e., $18.2 \%$ of all admissions for ICH. DOACs were the anticoagulants administered at admission in 9.5\% of cases of OAC-induced ICH. Patients in the ICH-DOAC group were older and more frequently had a previous ischemic stroke, lower ICH volume at admission, and lower stroke-related mortality (all differences statistically significant).

The EPICES study (a prospective study of patients with stroke admitted to the neurology department based on registries of data collected over 4 nonconsecutive months between 2008 and 2009 throughout Spain) showed that anticoagulant-associated ICH accounted for $8.4 \%$ of all brain hemorrhages [18]; this percentage was $13.6 \%$ in the TAC Registry study [3]. Part of the increased percentage in our study could be due to better control of arterial hypertension, which could have helped to reduce the incidence of the main cause of spontaneous ICH [19]. Nevertheless, global incidence in our study was greater than previously described (2.9 per 100,000 inhabitants/year in the TAC Registry 1 study), possibly due in part to the increase in the incidence of AF coinciding with aging of the population or prescription of anticoagulants.

Although up to $18 \%$ of patients aged $>80$ years in Spain have AF, the latest populationbased studies place the overall prevalence of $\mathrm{AF}$ in Spain at 4.4\%, a percentage that has remained stable since 1999 [20]. In the TAC Registry 2 study, we included patients receiving DOACs and not only those receiving VKA, as was the case in the TAC Registry 1 study. Therefore, given the results of pivotal clinical trials with DOACs, increasingly more patients with previously known AF may not have been receiving anticoagulant therapy because of the high risk of hemorrhage and are now receiving DOACs.

Observational studies $[21,22]$ showed that ICH-DOACs had a greater previous risk of hemorrhage than ICH-VKAs. In our study, both age and frequency of previous ischemic stroke were higher in the ICH-DOAC group. Both factors are included in bleeding risk scales, as are arterial hypertension, history of ICH, and leukoaraiosis, which in our study were more frequent - albeit not statistically significantly - in ICH-DOACs [23].

Nevertheless, almost $10 \%$ of OAC-induced ICH in our study and up to $20 \%$ in published series [4] were in patients taking DOACs; therefore, the risk of hemorrhage associated with these drugs, while significantly lower, cannot be underestimated. When DOACs were first introduced, they were thought to have 2 main disadvantages: the absence of a monitoring parameter and the absence of an antidote to reverse their effect [15]. First, of note, only 1 in 3 ICH-VKAs (28.7\% in our study) occur when the INR is supratherapeutic; therefore, a monitoring method is not necessarily valid for predicting the risk of bleeding. Second, although VKAs have a specific antidote, the effect takes hours to appear; in addition, other treatments, such as prothrombin complex and fresh frozen plasma have been proven to rapidly correct INR but were not associated with a lower mortality rate [6, 24, 25]. Furthermore, monoclonal antibodies that can reverse the anticoagulant effect of DOACs have been developed in recent years [26, 27], although none of the patients in the present study received these drugs; therefore, we were unable to evaluate their usefulness.

Other factors that affect the prognosis of ICH include age, infratentorial location, and Glasgow scale score on admission [28]. Hematoma growth is also a key determinant of prognosis and depends in turn on the initial ICH volume, recurrence of ICH, previous anticoagulation therapy with VKAs, and extension to the ventricles [29]. Therefore, in our study, it was noteworthy that in ICH-DOACs, the bleed was smaller, an intraventricular component was less frequent, and stroke-related mortality was lower than in ICH-VKAs (all differences statistically significant). In other studies [4, 21, 30], there was also a greater trend toward functional independence at 3 months, which in our study, as in a recent study in Portugal [22], did not reach statistical significance. 
Large observational studies have shown that the risk of thromboembolic events is greater in patients who do not restart anticoagulation therapy and that restarting treatment after the favorable outcome of the previous ICH is confirmed does not increase the risk of death [3133]. Some authors believe that anticoagulation therapy is commonly recommended after ICH, with DOACs as the treatment of choice owing to their safety profile [34]. Under these circumstances, there is no evidence for greater safety with one DOAC over another; therefore, this decision, which in our series favors apixaban, seems to depend on individual factors $[14,35]$.

In most cases, given that the combined risk of recurrence of ICH and stroke seems to be lowest at some time between week 1 and week 30 [27, 36], the most recent clinical practice guidelines recommend restarting anticoagulation therapy between weeks 4 and 8, especially when the cause of bleeding has been resolved [34]. In the present study, $18.89 \%$ of patients were receiving antiplatelet treatment and $16.59 \%$ were not receiving treatment 3 months after their ICH, possibly reflecting the lack of adherence to guidelines.

Our study is subject to a series of limitations. The fact that it is a retrospective study based only on data from patients attended in the neurology departments of the Spanish National Health System could have led to underrecording. Furthermore, for each patient, we only have neuroimaging findings and laboratory findings at admission; therefore, we were unable to evaluate the effect of therapy on these surrogate parameters and the possible association with morbidity and mortality. Additionally, different investigators measured ICH volume. Lastly, in several patients, we do not know the functional prognosis or prophylaxis prescribed.

In summary, we found that the incidence of OAC-induced ICH is greater than that described elsewhere and that it has tended to rise in recent years in Spain, although with slightly lower mortality. Hematoma volume and the frequency of ICH-related death were lower in patients who had previously received treatment with DOACs; therefore, the results of our study indicate that these drugs are the safest option in patients with nonvalvular AF and a high risk of hemorrhage.

\section{Acknowledgments}

Writing assistance was provided by Content Ed Net, Madrid, Spain.

\section{Statement of Ethics}

As this was a noninterventional retrospective study in which patients' personal data were not collected, informed consent was not necessary. The study was approved by the Ethics Committee of Hospital Universitario de La Princesa, Madrid, Spain, and a request for the study to be classified was made to the Spanish Agency of Medicinal Products and Medical Devices, which approved the classification of postauthorization study.

\section{Disclosure Statement}

The authors declare that they have no conflicts of interest.

\section{Funding Sources}

This study was partly funded by a local grant from Bristol-Myers Squibb/Pfizer. 


\section{Appendix}

\section{Study Investigators}

\begin{tabular}{|c|c|c|}
\hline $\begin{array}{l}\text { Autonomous } \\
\text { Community }\end{array}$ & Hospital & Investigator(s) \\
\hline Andalucía & Virgen del Rocío, Sevilla & $\begin{array}{l}\text { Francisco Moniche, Blanca Pardo-Galiana, Irene } \\
\text { Escudero }\end{array}$ \\
\hline Aragón & Clínico Universitario Lozano Blesa, Zaragoza & Carlos Tejero \\
\hline Asturias & Central de Oviedo, Asturias & Sergio Calleja, Lorena Benavente \\
\hline Balearic Islands & Son Espases, Baleares & Carmen Jiménez Martínez, María Moragues \\
\hline \multirow[t]{3}{*}{ Castilla La Mancha } & $\begin{array}{l}\text { Complejo Hospitalario General Universitario de } \\
\text { Albacete }\end{array}$ & Eva Fernández Díaz, Ana López, Tomás Segura \\
\hline & Hospital General La Macha Centro, Ciudad Real & Enrique Botia, Pablo del Saz, Rafael García Ruiz \\
\hline & Complejo Hospitalario de Toledo & Carlos Marsal, Francisco Muñoz \\
\hline \multirow[t]{6}{*}{ Catalonia } & Hospital del Mar, Barcelona & Jaume Roquer, Alejandra Gomez Gonzalez \\
\hline & Hospital Universitari Clínic de Barcelona & Angel Chamorro, Blanca Llull \\
\hline & $\begin{array}{l}\text { Hospital Universitari de Bellvitge, L’Hospitalet de } \\
\text { Llobregat }\end{array}$ & Pere Cardona \\
\hline & Santa Creu i Sant Pau, Barcelona & Joan Martí Fabregas, Alejandro Martínez Domeño \\
\hline & Arnau de Vilanova, Lleida & Francisco Purroy \\
\hline & Dr. Josep Trueta. Girona & Joaquín Serena \\
\hline \multirow[t]{3}{*}{ Galicia } & Complejo Hospitalario Universitario A Coruña & $\begin{array}{l}\text { Mar Castellanos, Alexia Roel García, María Feal Pain- } \\
\text { ceiras. }\end{array}$ \\
\hline & $\begin{array}{l}\text { Hospital Clínico Universitario de Santiago de } \\
\text { Compostela }\end{array}$ & Manuel Rodríguez Yáñez \\
\hline & Complexo Hospitalario Universitario de Vigo & José Maciñeiras, Pablo Vicente Alba \\
\hline \multirow[t]{2}{*}{ Canary Islands } & Hospital Ntra. Sra. De la Candelaria, Tenerife & Antonio Medina \\
\hline & $\begin{array}{l}\text { Hospital Universitario de Gran Canaria Dr Negrín, Las } \\
\text { Palmas de Gran Canaria }\end{array}$ & Juan Carlos López Fernández, Diana Ramos Rodríguez \\
\hline \multirow[t]{6}{*}{ Madrid } & Clínico Universitario San Carlos, Madrid & José Egido \\
\hline & Universitario de La Princesa, Madrid & $\begin{array}{l}\text { Gustavo Zapata Wainberg, Sonia Quintas Gutiérrez, } \\
\text { Álvaro Ximénez-Carrillo, José Vivancos }\end{array}$ \\
\hline & Universitario La Paz, Madrid & Blanca Fuentes \\
\hline & Universitario Ramón y Cajal, Madrid & Jaime Masjuán \\
\hline & General Universitario Gregorio Marañón, Madrid & $\begin{array}{l}\text { Andres García Pastor, Sergio López Anguita, Ana } \\
\text { Saldaña Díaz }\end{array}$ \\
\hline & Universitario 12 de Octubre, Madrid & Jaime Díaz Guzmán \\
\hline Murcia & Universitario Virgen de la Arrixaca, Murcia & Laura Albert \\
\hline \multirow[t]{2}{*}{ Basque Country } & Basurto, Bilbao & Mar Freijó \\
\hline & Universitario de Donosti & Maite Martínez, Amaia Muñoz Lopetegui \\
\hline \multirow[t]{2}{*}{ Valencia } & Universitario y Politécnico La Fe, Valencia & Aida Lago \\
\hline & Clínico Universitario de Valencia & José Miguel Laínez, Anna Ramos \\
\hline
\end{tabular}




\section{References}

1 van Nieuwenhuizen KM, van der Worp HB, Algra A, et al: Apixaban versus antiplatelet drugs or no antithrombotic drugs after anticoagulation-associated intraCerebral HaEmorrhage in patients with Atrial Fibrillation (APACHE-AF): study protocol for a randomised controlled trial. Trials 2015;16:393.

2 Milling TJ, Spyropoulos AC: Re-initiation of dabigatran and direct factor Xa antagonists after a major bleed. Am J Med 2016;129:S54-S63.

3 Zapata-Wainberg G, Ximénez-Carrillo Rico Á, Benavente Fernández L, et al: Epidemiology of intracranial haemorrhages associated with vitamin K antagonist oral anticoagulants in Spain: TAC Registry. Interv Neurol 2015;4:52-58.

4 Wilson D, Charidimou A, Shakeshaft C, et al: Volume and functional outcome of intracerebral hemorrhage according to oral anticoagulant type. Neurology 2015;86:360-366.

5 Flaherty ML, Tao H, Haverbusch M, et al: Warfarin use leads to larger intracerebral hematomas. Neurology 2008;71:1084-1089.

6 Steiner T, Rosand J, Diringer M: Intracerebral hemorrhage associated with oral anticoagulant therapy: current practices and unresolved questions. Stroke 2006;37:256-262.

7 Sjöblom L, Hårdemark HG, Lindgren A, et al: Management and prognostic features of intracerebral hemorrhage during anticoagulant therapy: a Swedish multicenter study. Stroke 2001;32:2567-2574.

8 Connolly SJ, Ezekowitz MD, Yusuf S, et al: Dabigatran versus warfarin in patients with atrial fibrillation. N Engl J Med 2009;361:1139-1151.

9 Patel MR, Mahaffey KW, Garg J, et al: Rivaroxaban versus warfarin in nonvalvular atrial fibrillation. N Engl J Med 2011;365:883-891.

10 Granger CB, Alexander JH, McMurray JJ, et al: Apixaban versus warfarin in patients with atrial fibrillation. N Engl J Med 2011;365:981-992.

11 Giugliano RP, Ruff CT, Braunwald E, et al: Edoxaban versus warfarin in patients with atrial fibrillation. N Engl J Med 2013;369:2093-2104.

12 Flaker GC, Eikelboom JW, Shestakovska, et al: Bleeding during treatment with aspirin versus apixaban in patients with atrial fibrillation unsuitable for warfarin: the apixaban versus acetylsalicylic acid to prevent stroke in atrial fibrillation patients who have failed or are unsuitable for vitamin $\mathrm{K}$ antagonist treatment (AVERROES) trial. Stroke 2012;43:3291-3297.

13 Ellis MH, Neuman T, Bitterman H, et al: Bleeding in patients with atrial fibrillation treated with dabigatran, rivaroxaban or warfarin: a retrospective population-based cohort study. Eur J Intern Med 2016;33:55-59.

14 Chatterjee S, Sardar P, Biondi-Zoccai G, Kumbhani DJ: New oral anticoagulants and the risk of intracranial hemorrhage: traditional and Bayesian meta-analysis and mixed treatment comparison of randomized trials of new oral anticoagulants in atrial fibrillation. JAMA Neurol 2013;70:1486-1490.

15 Zapata Wainberg G, Ximénez-Carrillo Rico A, Vivancos Mora J: Manejo clínico de los nuevos anticoagulantes. Neurología 2012;27:33-38.

16 R Development Core Team. R: a Language and Environment for Statistical Computing. Vienna, R Foundation for Statistical Computing, 2008.

17 Sullivan KM, Dean A, Soe MM: OpenEpi: a web-based epidemiologic and statistical calculator for public health. Public Health Rep 1989;124:471-474.

18 Arias-Rivas S, Vivancos J, Castillo J, et al: Epidemiologia de los subtipos de ictus en pacientes hospitalizados atendidos por neurólogos;resultados del registro EPICES (I). Rev Neurol 2012;54:385-393.

19 An SJ, Kim TJ, Yoon B: Epidemiology, risk factors, and clinical features of intracerebral hemorrhage: an update. J Stroke 2017;19:3-10.

20 Gómez-Doblas JJ, Muñiz J, Martin JJ, et al: Prevalence of atrial fibrillation in Spain. OFRECE study results. Rev Esp Cardiol (Engl Ed) 2014;67:259-269.

21 Hagii J, Tomita H, Metoki N, et al: Characteristics of intracerebral hemorrhage during rivaroxaban treatment: comparison with those during warfarin. Stroke 2014;45:2805-2807.

22 Marques-Matos C, Alves JN, Marto JP, et al: POST-NOAC: Portuguese observational study of intracranial hemorrhage on non-vitamin K antagonist oral anticoagulants. Int J Stroke 2017;12:623-627.

23 Pisters R, Lane DA, Nieuwlaat R, de Vos CB, Crijns HJ, Lip GY: A novel user-friendly score (HAS-BLED) to assess 1-year risk of major bleeding in patients with atrial fibrillation: the Euro Heart Survey. Chest 2010;138:10931100.

24 Dowlatshahi D, Butcher KS, Asdaghi N, et al: Poor prognosis in warfarin-associated intracranial hemorrhage despite anticoagulation reversal. Stroke 2012;43:1812-1817.

25 Parry-Jones AR, Di Napoli M, Goldstein JN, et al: Reversal strategies for vitamin K antagonists in acute intracerebral hemorrhage. Ann Neurol 2015;78:54-62.

26 Pollack CV Jr, Reilly PA, Eikelboom J, et al: Idarucizumab for dabigatran reversal. N Engl J Med 2015;373:511520.

27 Connolly SJ, Milling TJ Jr, Eikelboom JW, et al: Andexanet alfa for acute major bleeding associated with factor Xa inhibitors. N Engl J Med 2016;375:1131-1141.

28 Hemphill JC 3rd, Bonovich DC, Besmertis L, Manley GT, Johnston SC: The ICH score: a simple, reliable grading scale for intracerebral hemorrhage. Stroke 2001;32:891-897. 
29 Wang X, Arima H, Al-Shahi Salman R, et al: Clinical prediction algorithm (BRAIN) to determine risk of hematoma growth in acute intracerebral hemorrhage. Stroke 2015;46:376-381.

30 Takahashi H, Jimbo Y, Takano H, et al: Intracerebral hematoma occurring during warfarin versus non-vitamin K antagonist oral anticoagulant therapy. Am J Cardiol 2016;118:222-225.

31 Nielsen PB, Larsen TB, Skjøth F, Lip GYH: Outcomes associated with resuming warfarin treatment after hemorrhagic stroke or traumatic intracranial hemorrhage in patients with atrial fibrillation. JAMA Intern Med 2017; 177:1-7.

32 Kuramatsu JB, Gerner ST, Schellinger PD, et al: Anticoagulant reversal, blood pressure levels, and anticoagulant resumption in patients with anticoagulation-related intracerebral hemorrhage. JAMA 2015;313:824836.

33 Yung D, Kapral MK, Asllani E, Fang J, Lee DS; Investigators of the Registry of the Canadian Stroke Network: Reinitiation of anticoagulation after warfarin-associated intracranial hemorrhage and mortality risk: the Best Practice for Reinitiating Anticoagulation Therapy after Intracranial Bleeding (BRAIN) Study. Can J Cardiol 2012;28:33-39.

34 Kirchhof P, Benussi S, Kotecha D, et al: 2016 ESC Guidelines for the management of atrial fibrillation developed in collaboration with EACTS. Eur J Cardiothorac Surg 2016;50:e1-e88.

35 Yoon CH, Park YK, Kim SJ, et al: Eligibility and preference of new oral anticoagulants in patients with atrial fibrillation: comparison between patients with versus without stroke. Stroke 2014;45:2983-2988.

36 Majeed A, Kim YK, Roberts RS, Holmström M, Schulman S: Optimal timing of resumption of warfarin after intracranial hemorrhage. Stroke 2010;41:2860-2866. 